\title{
Resultados en salud y eficiencia del fentanilo intranasal en pectina en el dolor irruptivo en la práctica clínica habitual
}

\author{
B. Blanco ${ }^{1}$, A. J. García Ruiz ${ }^{2}$ y N. García-Agua Soler ${ }^{2}$ \\ ${ }^{1}$ Hospital de Elda. ${ }^{2}$ Cátedra de Economía de la Salud y Uso Racional del Medicamento. Departamento de \\ Farmacología. Facultad de Medicina. Universidad de Málaga.
}

B. Blanco, A. J. García Ruiz y N. García-Agua Soler. Resultados en salud y eficiencia del fentanilo intranasal en pectina en el dolor irruptivo en la práctica clínica habitual. Rev Soc Esp Dolor 2013; 20(5): 221-229.

\begin{abstract}
Objective: Determine, with a pharmacoeconomic evaluation, the effectiveness and efficiency of fentanyl pectin intranasal spray for BTPc versus other indicated treatments.

Results and conclusions: All the treatments analyzed in the pharmacoeconomic evaluation were cost-effective in terms of a willingness to pay (invest) of $€ 30,000$ per AVAC gained (cost / effectiveness ratio $<€ 30,000$ ). The most efficient option (greater effectiveness and lower cost) of all the treatments analyzed was the fentanyl pectin nasal spray (FPNS).

In the incremental analysis FPNS was the dominant treatment option over all the others evaluated: FST, OTFC, FBT and INFS.

From the analysis of the data of our cost/effectiveness modeling study of the fentanyl pectin nasal spray, FPNS, we can conclude:

- INFS without providing greater effectiveness has been shown to be significantly more expensive (+15\%).

- FST, OTFC, FBT have demonstrated $-18 \%$ less effectiveness and also been shown to be significantly more expensive than FPNS (+9\%; $+15 \%$; $+10 \%$, respectively).

Abbreviations: AVAC: Years gained with good quality of life. FPNS: PecFent ${ }^{\circledR}$. FST: Abstral ${ }^{\circledR}$. OTFC: Actiq ${ }^{\circledR}$. FBT: Effentora ${ }^{\circledR}$. INFS: Instanyl ${ }^{\circledR}$.
\end{abstract}

Key words: BreakThrough Cancer Pain. Fentanilo. Pharmacoeconomic. Cost-effectiveness.

Recibido: 01-08-13.

Aceptado: 15-11-13.

\section{RESUMEN}

Objetivo: Conocer la efectividad y eficiencia del fentanilo intranasal en pectina comparado con otros tratamientos empleados en el dolor irruptivo oncológico a través de un análisis farmacoeconómico.

Resultados y conclusiones: En la evaluación farmacoeconómica realizada, todas las opciones analizadas fueron coste-efectivas para una disposición a pagar (invertir) de $30.000 €$ por AVAC ganado (ratio coste/efectividad $<30.000 €$ ). La opción más eficiente (mayor efectividad y menor coste) en todos los casos analizados es el espray intranasal de fentanilo en pectina (FPNS).

En el análisis incremental, FPNS fue la opción dominante sobre todas las demás evaluadas: FST, OTFC, FBT e INFS.

Del análisis de los datos de nuestro estudio de modelización de coste/efectividad del espray intranasal de fentanilo en pectina FPNS se concluye:

- INFS sin proporcionar más eficacia ha demostrado ser significativamente más caro (+15\%).

- FST, OTFC, FBT han demostrado una efectividad un $18 \%$ menor que FPNS y además ser significativamente más caros (+9\%; +15\%; +10\%, respectivamente).

Abreviaturas: AVAC: años ganados con buena calidad de vida. FPNS: PecFent ${ }^{\circledR}$. FST: Abstral ${ }^{\circledR}$. OTFC: Actiq ${ }^{\circledR}$. FBT: Effentora ${ }^{\circledR}$. INFS: Instany ${ }^{\circledR}$.

Palabras clave: Dolor irruptivo. Fentanilo. Farmacoeconomía. Eficiencia.

\section{INTRODUCCIÓN}

El dolor irruptivo oncológico (DIO) se define como una "exacerbación transitoria del dolor que aparece, ya sea de forma espontánea o bien relacionada con un desencadenan- 
te concreto, predecible o impredecible, a pesar de existir un dolor basal estable y adecuadamente controlado" (1). Es un factor predictivo de mal pronóstico en el control del dolor $(2,3)$ con un importante impacto negativo en la calidad de vida de los pacientes que lo sufren.

Su prevalencia varía según las series estudiadas (33-95 \%), pero podemos resumir que aumenta a medida que la enfermedad progresa, empeora el estado funcional y se ven afectados por el tumor estructuras óseas y plexos (4).

El DIO se caracteriza por alcanzar el pico de dolor en los primeros 5 minutos (5), intensidad severa, duración breve ( $<60 \mathrm{~min}$ ) con una media de ocurrencia de 3-5 episodios al día (6).

A pesar del interés creciente en los últimos años por esta entidad y el desarrollo de estrategias terapéuticas (fentanilos transmucoso e intranasales) que mimeticen su patrón temporal y ser los fentanilos considerados el tratamiento de elección (7), el DIO sigue estando infradiagnosticado y no siempre adecuadamente tratado. En un estudio reciente publicado por Davies et al. en el cual se incluyeron 1.000 pacientes de 13 países europeos con DIO, aproximadamente el $50 \%$ reconocía que no tomaba el opioide prescrito en cada episodio de DIO y las razones, entre otras, eran la falta de respuesta al fármaco prescrito. Al analizar el opioide de rescate de la muestra se observó que menos del $20 \%$ tenían prescrito un fentanilo transmucoso para su tratamiento (6).

La optimización del control sintomático en el paciente oncológico con una pauta analgésica adecuada no solo tendrá repercusión en la mejora de su calidad de vida y funcionalidad sino también en los costes indirectos asociados (menos visitas a urgencias, menos consultas no programadas y menor número de ingresos hospitalarios).

En un análisis retrospectivo llevado a cabo en el Servicio de Cuidados Paliativos del Hospital General Universitario de Elda, se analizaron 47 pacientes que habían sido derivados a dicho servicio desde otros servicios hospitalarios y presentaban DIO. El $40 \%$ de la muestra, previo a su ingreso en el Servicio de Cuidados Paliativos, tenía pautado para el tratamiento del DIO un opioide de acción corta (morfina, oxicodona), otro $45 \%$ tenía un opioide de liberación inmediata (fentanilo transmucoso) y el restante $15 \%$ un fármaco de primer escalón o sencillamente no tenían nada prescrito. De los 47 pacientes, 43 llevaban tratamiento para el dolor basal (los pacientes restantes no lo precisaban). Hubo que realizar ajuste del tratamiento para el dolor basal en 34 de los 43 pacientes (en 29 se subió y en 5 pacientes se bajó el tratamiento). En los restantes 9 pacientes se mantuvo la dosis para el tratamiento del dolor basal. En la recogida retrospectiva se analizaron los pacientes en los que tras la primera valoración, se cambió el opioide de rescate a fentanilo intranasal en pectina y se procedió a su ajuste de dosis.

El seguimiento medio fue de 28,11 días (DE 15,39). En la mayoría de los pacientes, $73 \%$, el tratamiento con fentanilo nasal en pectina fue bien tolerado (el restante $27 \%$ presentó estreñimiento moderado, si bien el $26 \%$ ya lo presentaba por el opioide basal). En el $100 \%$ se consiguió un buen ajuste de dosis, que consiguió una disminución de la intensidad del dolor en crisis antes-después de 7 (DE 1,06) vs. 3,89 (DE 0,77) medido mediante una escala numérico-verbal de 0 a 10.

La calidad de vida relacionada con la salud (medida con la EORTC-QLQ-C30) también mejoró de forma significativa tras alcanzar la mejora analgésica.

El análisis pre-post también demostró una disminución en el número de ingresos hospitalarios por paciente (155 pre. vs. 12 post. ingresos hospitalarios) y, en consecuencia, una disminución de los costes asociados (857 pre. vs. 15 post. días de ingreso).

La optimización del control sintomático y analgésico es, sin duda, una de las causas del menor consumo de recursos sanitarios (8). La situación económica global nos obliga al uso racional de dichos recursos y a buscar no solo la eficacia sino también la eficiencia en nuestra práctica clínica.

Estos resultados del Servicio de Cuidados Paliativos del Hospital General Universitario de Elda despertaron el interés por conocer la efectividad y eficiencia del fentanilo intranasal en pectina comparado con otros tratamientos empleados en el DIO, objetivo del presente estudio farmacoeconómico.

\section{OBJETIVO}

Conocer la efectividad y eficiencia del fentanilo intranasal en pectina comparado con otros tratamientos empleados en el dolor irruptivo a través de un metaanálisis de sus más importantes publicaciones.

\section{METODOLOGÍA EMPLEADA}

Se ha realizado una doble evaluación clínica y económica de los medicamentos con indicación en el dolor irruptivo de cáncer en nuestro país (BreakThrough cancer pain $B T C P)$. De una parte, se han evaluado los medicamentos desde la eficacia a partir de las fichas técnicas de cada uno de ellos y de un metaanálisis.

Para apoyar en la toma de decisiones, se ha desarrollado un modelo de análisis que estima el coste-utilidad (coste/ QALYs) de los medicamentos estudiados. Este modelo analiza las reducciones previstas en la intensidad del dolor (PI) de los episodios de BTPC y cómo se tradujeron en ahorros de costes, la utilidad se midió en el número de años ganados con buena calidad de vida (AVAC).

Los medicamentos analizados fueron los diversos compuestos de fentanilo con indicación aprobada en nuestro país para dolor irruptivo por diferentes vías a excepción 
de la parenteral: comprimidos sublinguales ( $F S T$ - fentanyl sublingual tablets - Abstral $\left.{ }^{\mathbb{}}\right)$; comprimidos para chupar con aplicador bucal integrado (OTFC - oral transтисоsal fentanyl citrate - Actiq $\left.{ }^{\circledR}\right)$; comprimidos bucales (FBT - fentanyl buccal tablet - Effentora ${ }^{\mathbb{R}}$ ); pulverización acuosa nasal (INFS - intra nasal fentanyl spray - Instanyl ${ }^{\mathbb{}}$ ); espray de fentanilo intranasal en pectina (FPNS - fentanyl pectin nasal spray - Pecfent ${ }^{\mathbb{R}}$.

La morfina de liberación rápida (IRMS - Sevredol ${ }^{\circledR} y$ $\left.\operatorname{Oramorph}^{\circledR}\right)$ fue excluida del análisis debido a que no tiene indicación para el tratamiento del dolor irruptivo (Breakthrough cancer pain - BTCP).

\section{Datos de eficacia}

Los resultados de eficacia de los medicamentos estudiados proceden de la revisión de las fichas técnicas de cada uno de los medicamentos estudiados (9) y de un metaanálisis de modelos de efectos fijos (10). Los resultados de todos los ensayos se analizaron simultáneamente con un método de comparación indirecta (mixed-treatment comparison $M T C$ ). Este método puede ser considerado como un método válido cuando los estudios incluidos son comparables con respecto a los pacientes, el efecto modificador de base y las características del estudio.

Los sujetos analizados incluidos en este metaanálisis eran pacientes con un dolor basal moderado $(\leq 4$, en una escala de 0-10) (11-13) antes del episodio de dolor irruptivo y controlados con tratamiento opioide.

Como hemos comentado, este artículo realiza un análisis coste/utilidad de los medicamentos estudiados (Abstral ${ }^{\mathbb{R}}$, Actiq $^{\circledR}$, Effentora $^{\circledR}$, Instanyl $l^{\circledR}$ y Pecfent $\left.^{\circledR}\right)$.

\section{Utilidad}

La utilidad, o parte cualitativa de este artículo, analiza la mejora en la calidad de vida de los pacientes medida en número de años ganados con buena calidad de vida (AVAC). Para ello se analizó la eficacia de los distintos fentanilos para reducir la intensidad del dolor y la eficacia se transformó en su utilidad o AVACs.

La eficacia del metaanálisis midió la reducción en la intensidad de dolor irruptivo superior o igual a 2 puntos en la escala (respecto del inicio), medido como disminución del dolor representado por el área bajo la curva (AUC) respecto a placebo. La transformación de eficacia en utilidad (años de vida ganados con buena calidad de calidad - AVACs o QALYs) fueron obtenidas a partir de un artículo (14) en el que se realizó un análisis de preferencias que revelaron las utilidades asignadas a cada estado de salud, estas se ajustaron mediante la siguiente ecuación de regresión lineal: Utilidad $=-0,1237^{*} A U C+0,9536 ; R 2=0,93$ (donde $\mathrm{AUC}$ = es la disminución del área bajo la curva del tratamiento respecto a placebo).

En otras palabras, los AVACs en este estudio indican los años adicionales que un paciente está dispuesto a vivir dada la calidad de vida que los distintos fármacos le proporcionan (por la mejora analgésica en sus episodios de dolor irruptivo).

En la Tabla I se muestran las utilidades empleadas en el análisis y el coste por tratamiento, así como sus intervalos de confianza necesarios para el análisis de sensibilidad.

\section{Coste}

El coste, o parte cuantitativa de este artículo, se corresponde a los costes de adquisición de la medicación empleada para el dolor irruptivo a PVP+IVA.

Se ha tomado como media en cada paciente 3 episodios de dolor irruptivo al día durante un máximo de 6 meses (180 días).

\section{Perspectiva del análisis y horizonte temporal}

La perspectiva de nuestro análisis es la del pagador (Sistema Nacional de Salud, extensible a las CC.AA. y los hospitales). La duración total del periodo analizado es de 180 días, por lo cual no hubo que realizar descuentos ni en los efectos ni en los costes.

\section{Estructura del modelo y diseño del estudio}

En nuestro estudio se han seguido las directrices generales para la realización de análisis farmacoeconómicos en España, de la Canadian Agency for Drugs and Technologies in Health (CADTH) y de la International Society for Pharmacoeconomics and Outcomes Research (ISPOR) (15-17).

El modelo de simulación de Monte Carlo (MCM) ha sido desarrollado para comparar la relación coste/eficacia (RCE) de 5 estrategias diferentes de tratamiento en el dolor irruptivo. El modelo se construyó utilizando el software TreeAge (@ 2009 TreeAge Software, Inc. - Decision Analysis Software). En la Figura 1 se muestra el modelo analizado.

Los parámetros de resultados incluidos en el modelo de Monte Carlo para cada medicamento estudiado fueron: porcentaje de episodios de dolor irruptivo controlados satisfactoriamente, coste de la medicación empleada, y años de vida ganados con buena calidad de la misma (AVACs ganados).

El modelo evaluó la relación coste-utilidad de cada medicamento usado como primer escalón para alcanzar el 
TABLA I. DATOS DE UTILIDAD, EFICACIA Y COSTES EMPLEADOS EN EL ANÁLISIS FARMACOECONÓMICO

\begin{tabular}{|c|c|c|c|}
\hline Medicamento analizado & $\begin{array}{l}\text { \% de episodios de dolor } \\
\text { irruptivo controlados } \\
\text { (IC95\%) (refs. } 9 \text { y 14) }\end{array}$ & $\begin{array}{c}\text { Años ganados con calidad } \\
\text { de vida (AVACs) } \\
\text { (IC95\%) (ref. 14) }\end{array}$ & $\begin{array}{c}\text { Coste de adquisición del } \\
\text { medicamento (180 dias) } \\
\text { (PVP+IVA) (ref. 9) }\end{array}$ \\
\hline $\begin{array}{l}\text { Comprimidos sublinguales } \\
\left(\text { Abstral }^{\mathbb{}}\right)\end{array}$ & \multirow{2}{*}{$\begin{array}{c}29 \% \\
(22 \%-38 \%)\end{array}$} & \multirow{2}{*}{$\begin{array}{c}0,220 \\
(0,203-0,235)\end{array}$} & $4.163 €$ \\
\hline $\begin{array}{l}\text { Comprimidos con } \\
\text { aplicador bucal }\left(\text { Actiq }^{\circledR}\right)\end{array}$ & & & $4.412 €$ \\
\hline $\begin{array}{l}\text { Comprimidos bucales } \\
\left(\text { Effentora }^{\circledR}\right)\end{array}$ & $\begin{array}{c}31 \% \\
(25 \%-39 \%)\end{array}$ & $\begin{array}{c}0,223 \\
(0,209-0,237)\end{array}$ & $4.222 €$ \\
\hline $\begin{array}{l}\text { Pulverización acuosa nasal } \\
\left(\text { Instanyl }{ }^{\circledR}\right)\end{array}$ & \multirow{2}{*}{$\begin{array}{c}55 \% \\
(46 \%-68 \%)\end{array}$} & $\begin{array}{c}0,266 \\
(0,251-0,281)\end{array}$ & $4.390 €$ \\
\hline $\begin{array}{l}\text { Espray intranasal de } \\
\text { fentanilo en pectina } \\
\left(\text { Pecfent }^{\circledR}\right)\end{array}$ & & $\begin{array}{c}0,245 \\
(0,221-0,268)\end{array}$ & $3.834 €$ \\
\hline
\end{tabular}

El fentanilo intranasal consigue controlar el mayor porcentaje de episodios de dolor irruptivo, en concreto un $55 \%$ vs. el $31 \%$ que consiguen controlar los comprimidos bucales y el $29 \%$ (los comprimidos con aplicador y los comprimidos sublinguales). El espray intranasal de fentanilo en pectina proporciona más años ganados con calidad de vida que las formas orales (sublingual, aplicador bucal o comprimido bucal) y además al menor coste de adquisición de todos.

Nota explicativa: $A V A C=$ años ganados con calidad de vida. Costes = costes de la medicación durante 180 dias con 3 episodios de dolor irruptivo por dia/paciente.

objetivo terapéutico. El resultado económico principal del modelo de MCM fue el coste por AVAC ganado. Dicho de otro modo, qué opción proporciona más años de vida ganados, con calidad, a menor coste.

Para cada medicamento analizado se calculó la ratio coste-efectividad y el coste-efectividad incremental (ICER) respecto de la opción más coste-efectiva, que se calcula como la diferencia de costes entre los 2 comparadores dividido por su diferencia en eficacia (utilidad): ICER $=($ coste farm. A - coste farm. B)/(efic. A - efic. B). En este estudio, se asumió que cualquier valor de ICER hasta $30.000 €$ es rentable en nuestro país (el estudio de Sacristán y cols. indica la disposición a invertir hasta $30.000 €$ por cada año de vida ganado) (18).

Para la modelización se consideró como caso de referencia un dolor basal de 2 en la escala de dolor. En los ensayos analizados en el metaanálisis, los episodios de dolor irruptivo oscilaron entre 1 y 4 al día (para nuestro caso base se consideró 3 episodios/día). También se supuso que todos los episodios tenían una duración de 1 hora. La mayoría de los pacientes con BTCP tiene cáncer en etapa avanzada, por lo cual un horizonte temporal de 1 año se supone que es adecuado, con una esperanza de vida de 180 días que se utiliza para el escenario base.

Los resultados de los análisis probabilísticos se presentan en forma de Curva de Aceptabilidad de Coste-Efectividad (CEACs).

\section{Análisis de sensibilidad}

En nuestro estudio se realizó un análisis de sensibilidad probabilístico que permite el análisis de la incertidumbre a través de múltiples parámetros de entrada que deben evaluarse. Las fuentes de incertidumbre de los parámetros fueron identificadas y caracterizadas como distribuciones de probabilidad (normal para los datos de costes y beta para las probabilidades de reducción del dolor - PI - y calidad de vida ganada - AVACs). Los resultados se presentan con una estimación puntual y el intervalo de confianza (IC $95 \%$ ) de los parámetros del modelo.

\section{Análisis estadístico}

El análisis estadístico se ha realizado con el paquete SPSS v20 licenciado para la Universidad de Málaga.

En la estadística descriptiva para las variables cualitativas se ha realizado la frecuencia y proporción. Para variables cuantitativas, media y desviación estándar e intervalo de confianza al $95 \%$.

Para conocer las diferencias entre ambos grupos de estudio (estadística inferencial), se han realizado los test de Chi-cuadrado, T-Student (para muestras relacionadas) y Anova de una vía. 


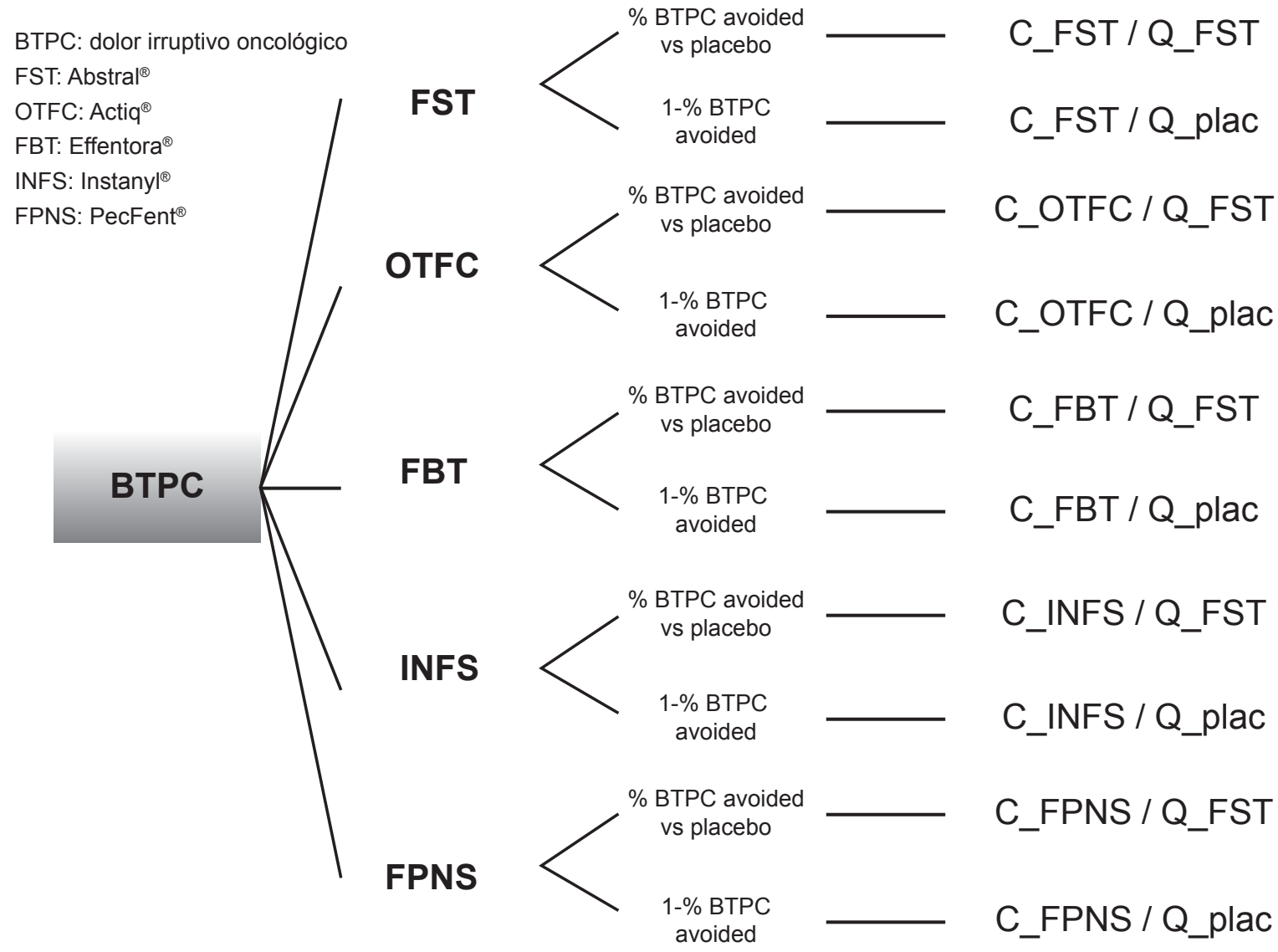

\% BTPC: hace referencia al \% de pacientes que disminuyen la intensidad y duración del dolor de forma significativa.

$\left(C_{-}\right)$: es el coste del tratamiento con la medicación analizada suponiendo 3 episodios de dolor irruptivo al día durante 6 meses.

$\left(Q_{-}\right)$: hace referencia a los años ganados con calidad de vida (AVACs o QALYs).

Fig. 1. Modelo empleado en el análisis farmacoeconómico de las diferentes presentaciones de fentanilo en el dolor irruptivo. Árbol de decisión.

\section{RESULTADOS}

\section{Datos de eficacia}

\section{Eficiencia: relación coste/efectividad (RCE)}

La mayor eficacia, medida como ganancia en calidad de vida, se obtuvo con la opción de fentanilo intranasal de fentanilo en pectina frente a las otras opciones analizadas. Esta opción (FPNS) fue en todos los casos más de un 15\% superior en número de años ganados con buena calidad de vida (AVACs ganados) respecto a las opciones orales.

Comparando uno a uno los otros tratamientos vs. FPNS, los comprimidos sublinguales (FST) proporcionaron menos años ganados: $-18 \%$, los comprimidos con aplicador bucal integrado (OTFC): $-18 \%$, y los comprimidos bucales (FBT): $-17 \%$ (Tabla II).
La opción de mayor coste (por 180 días de tratamiento) tras el análisis farmacoeconómico fue para OTFC con $4.412 €$, seguida de INFS, FBT y FST (4.395€, $4.215 €$ y $4.160 €$, respectivamente). La opción más económica fue para FPNS con $3.824 €$ (Tabla II).

A partir de los AVACs conseguidos con seis meses de medicación se calculó la inversión necesaria para ganar un año de vida con calidad con los distintos tratamientos. Ver columna Coste/eficacia (€/AVACs) de la Tabla II.

De todas las opciones analizadas, la opción más eficiente resultó ser FPNS (mayor eficacia, más años ganados con buena calidad de vida a un menor coste). Todas las demás opciones fueron estrategias dominadas, es decir, presentaron una menor eficacia (FST, OTFC y FBT) o similar eficacia (INFS) presentando todas ellas un mayor coste. En la Tabla II se muestran los datos relativos a la evaluación farmacoeconómica realizada. 
TABLA II. RESULTADOS DEL ANÁLISIS FARMACOECONÓMICO. COSTES, EFICACIA Y RELACIÓN COSTE / EFICACIA INCREMENTAL (ICER) DE LAS OPCIONES ANALIZADAS PARA TRATAR EL DOLOR IRRUPTIVO EN ESPAÑA. SIMULACIÓN MONTE CARLO SOBRE 10.000 PACIENTES.

\begin{tabular}{|c|c|c|c|c|c|c|}
\hline \multirow[b]{2}{*}{ Estrategia } & \multicolumn{2}{|c|}{ Media por paciente } & \multirow{2}{*}{$\begin{array}{c}\text { Costeleficacia } \\
(€ / A V A C s)\end{array}$} & \multicolumn{2}{|c|}{ Diferencia respecto a FPNS } & \multirow[b]{2}{*}{ ICER } \\
\hline & $\begin{array}{c}\text { Costes }(€) \\
\text { medicación }\end{array}$ & $\begin{array}{l}\text { Eficacia } \\
(A V A C s)\end{array}$ & & $\begin{array}{c}\text { Costes }(€) \\
\text { medicación }\end{array}$ & $A V A C s$ & \\
\hline $\begin{array}{l}\text { Espray } \\
\text { intranasal } \\
\text { de fentanilo } \\
\text { en pectina } \\
\text { (FPNS, } \\
\left.\text { Pecfent }^{\mathbb{B}}\right)\end{array}$ & $3.824 €$ & 0,22136 & $17.275 €$ & - & - & $\begin{array}{c}\text { Opción más } \\
\text { eficiente }\end{array}$ \\
\hline $\begin{array}{l}\text { Comprimidos } \\
\text { sublinguales } \\
(F S T, \\
\left.\text { Abstral }^{\circledR}\right)\end{array}$ & $4.160 €$ & 0,18238 & $22.809 €$ & $\begin{array}{l}+336 € \\
(+9 \%)\end{array}$ & $\begin{array}{c}-0,039 \\
(-18 \%)\end{array}$ & $\begin{array}{l}\text { Opción } \\
\text { dominada }\end{array}$ \\
\hline $\begin{array}{l}\text { Comp. con } \\
\text { aplicador } \\
\text { bucal (OTFC, } \\
\left.\text { Actiq }^{\circledR}\right)\end{array}$ & $4.412 €$ & 0,18247 & $24.179 €$ & $\begin{array}{l}+588 € \\
(+15 \%)\end{array}$ & $\begin{array}{c}-0,039 \\
(-18 \%)\end{array}$ & $\begin{array}{c}\text { Opción } \\
\text { dominada }\end{array}$ \\
\hline $\begin{array}{l}\text { Comprimidos } \\
\text { bucales (FBT, } \\
\left.\text { Effentora }^{\circledR}\right)\end{array}$ & $4.215 €$ & 0,18438 & $22.860 €$ & $\begin{array}{l}+391 € \\
(+10 \%)\end{array}$ & $\begin{array}{c}-0,037 \\
(-17 \%)\end{array}$ & $\begin{array}{l}\text { Opción } \\
\text { dominada }\end{array}$ \\
\hline $\begin{array}{l}\text { Pulverización } \\
\text { acuosa nasal } \\
\text { (INFS, } \\
\left.\text { Instany } l^{\circledR}\right)\end{array}$ & $4.395 €$ & 0,22145 & $19.846 €$ & $\begin{array}{l}+571 € \\
(+15 \%)\end{array}$ & $\begin{array}{c}+0,0001 \\
(0 \%)\end{array}$ & $\begin{array}{c}\text { Opción } \\
\text { dominada }\end{array}$ \\
\hline
\end{tabular}

Ganar un año de vida con calidad se consigue con la menor inversión con el espray intranasal de fentanilo en pectina (FPNS), 17.275 €. En consecuencia, el FPNS proporciona el mejor ratio costeleficacia.

\begin{abstract}
Nota explicativa: Tanto los costes como las utilidades (AVACs) pueden cambiar respecto de la tabla I al emplearse un modelo probabilístico de análisis farmacoeconómico.

AVAC: años ganados con calidad de vida.
\end{abstract}

El mejor ratio coste/efectividad (RCE) fue para FPNS con 17.275 €/AVAC (opción más coste-efectiva), seguida de INFS (19.846 €/AVAC) y los peores ratios correspondieron a OTFC, FBT y FST $(24.179 €, 22.860 €$, y 22.809 $€ /$ AVAC, respectivamente).

En la Figura 2 se muestra la probabilidad de que las opciones de tratamiento sean más rentables, a diferentes niveles de disposición a invertir por un año ganado con calidad de vida (en la gráfica se analiza una posible inversión de 0 a $30.000 €)$. FPNS fue el tratamiento con mayor probabilidad de ser más coste-efectivo a cualquier inversión entre 0 y $30.000 € / A V A C$. En concreto, su probabilidad de ser el tratamiento más rentable osciló entre el $40 \%$ (para $0 €$ de disposición a invertir) y el $75 \%$ (para los $30.000 €$ de disposición a invertir).

\section{Coste-efectividad incremental (ICER)}

En la Figura 3 se muestra el coste/utilidad (eficacia) de las opciones estudiadas. Se puede observar como la opción más efectiva (más años ganados con calidad de vida) y menos costosa fue el FPNS. Tres de las demás opciones comparadas son inferiores en eficacia y todas presentan un mayor coste. Por tanto, la opción más eficiente es el FPNS.

\section{Análisis de sensibilidad probabilístico}

Para el análisis de sensibilidad probabilístico se tuvo en cuenta los valores medios, desviación estándar e intervalos de confianza de los costes, de las probabilidades de 


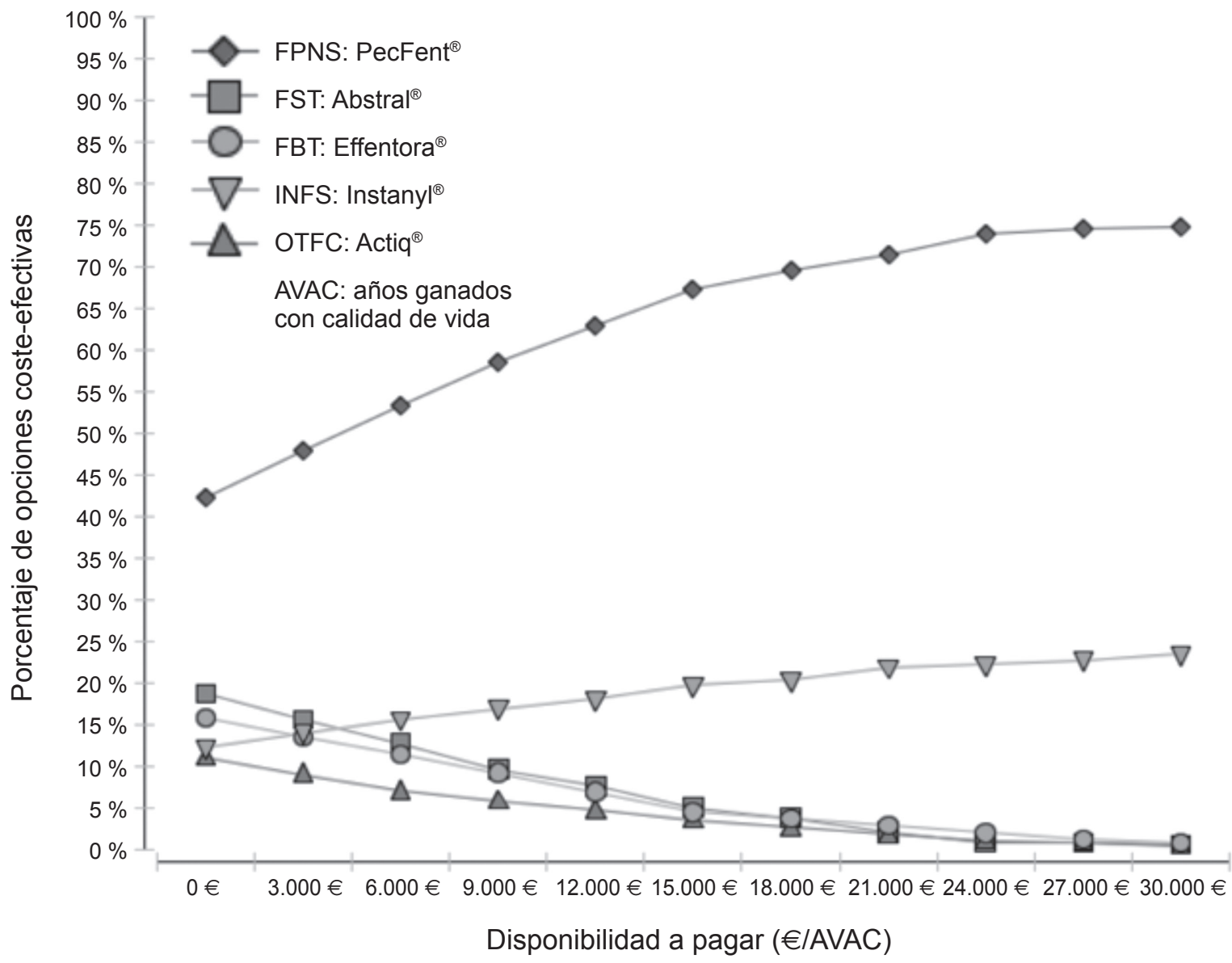

Sea cual sea la cantidad que estuviéramos dispuestos a invertir (de 0 a $30.000 €$ ) por ganar un año con calidad de vida, el espray intranasal de fentanilo en pectina (FPNS) demostró mayor probabilidad de ser el tratamiento más coste-efectivo. En concreto, desde un $40 \%$ de efectividad (para $0 €$ ) hasta un $75 \%$ (para $30.000 €$ ).

Fig. 2. Curva de aceptabilidad del coste eficacia de los tratamientos farmacológicos empleados en el tratamiento del dolor irruptivo en cáncer.

mejora en la intensidad del dolor de cada paciente y de la efectividad medida como años de vida ganados ajustados por calidad (Tabla I).

En todos los casos, FPNS fue la opción con mejor relación coste-efectividad comparada con las otras alternativas terapéuticas.

\section{DISCUSIÓN}

El médico, en su práctica clínica habitual debe decidir qué tratamiento es más coste-efectivo para su paciente. La calidad de la prescripción en terapéutica farmacológica implica que, desde un punto de vista clínico, el tratamiento farmacológico sea efectivo al menor coste posible; por ello, para evaluar la calidad de la prescripción y ser eficientes es conveniente distinguir entre aspectos de racionalidad farmacológica (eficacia del medicamento) y racionalidad económica (eficiencia del fármaco). Es importante mantener la eficacia cuidando la eficiencia, los recursos bien utilizados redundarán en beneficio de otros pacientes, que podrían necesitarlos.

La idea de la evaluación económica de los medicamentos debe pasar de ser un medio para la contención del gasto farmacéutico a ser una herramienta que contribuya decisivamente a la utilización más racional de estos, y mejorar la utilización de los recursos disponibles. Las autoridades sanitarias son las primeras interesadas en promover este tipo de evaluaciones, así como abrir los cauces para el establecimiento de una metodología que produzca análisis homogéneos y con rigor científico que sirva de apoyo al colectivo médico para la toma de decisiones posteriores (19).

En nuestro estudio se pone de manifiesto que conocer la eficiencia de los tratamientos puede ayudar al médico a realizar un uso más racional de los medicamentos, ya que puede conseguir más eficacia a un menor coste utilizando 
una combinación frente a otra, lo que en definitiva redundará en mayores beneficios tanto clínicos (para el paciente) como económicos (para la sociedad).

El dolor irruptivo, como sabemos, se asocia con un aumento del uso de los servicios sanitarios (más visitas a la consulta externa e ingresos hospitalarios) (20) y, por tanto, también aumentan los gastos directos (gastos en medicamentos) e indirectos (costes de transporte), para los servicios sanitarios, el paciente y sus cuidadores (21). El dolor irruptivo también se asocia con un aumento en el uso de los servicios sociales: puede influir en la capacidad laboral del paciente y de su cuidador (y, por tanto, en sus fuentes de ingresos). Además existen otros costes más amplios para la sociedad, como la pérdida de productividad (22).

Es muy importante sopesar el coste de cualquier intervención con el coste total de no intervenir (el coste total de no tratar el dolor irruptivo). Por ejemplo, se ha observado que el tratamiento con fentanilo transmucosa permite reducir el uso de ciertos servicios sanitarios (por ejemplo, ingresos hospitalarios) (23) y mejora la capacidad para trabajar/obtener ingresos (dolor no oncológico) (24).

\section{Eficiencia como relación coste/eficacia}

Como se ha muestra en nuestro trabajo, para mantener la misma eficiencia que el espray intranasal de fentanilo en pectina (FPNS), el fentanilo acuoso tendría que disminuir significativamente su precio y, aun así, no proporcionaría mayor eficacia, y los diferentes compuestos de fentanilo orales (sublingual, bucal) deberían aumentar la efectividad alrededor de un $20 \%$ a la que poseen y disminuir significativamente su precio (Tabla II, Figura 3 ).

El espray intranasal de fentanilo en pectina (FPNS) ofreció mayor beneficio neto (tanto en incremento de salud como monetario) frente a sus alternativas a cualquier disponibilidad a invertir (de 0 a $30.000 €$ ) por año ganado con calidad de vida (AVAC).

\section{CONCLUSIONES}

En la evaluación farmacoeconómica realizada todas las opciones analizadas fueron coste-efectivas para una dis-

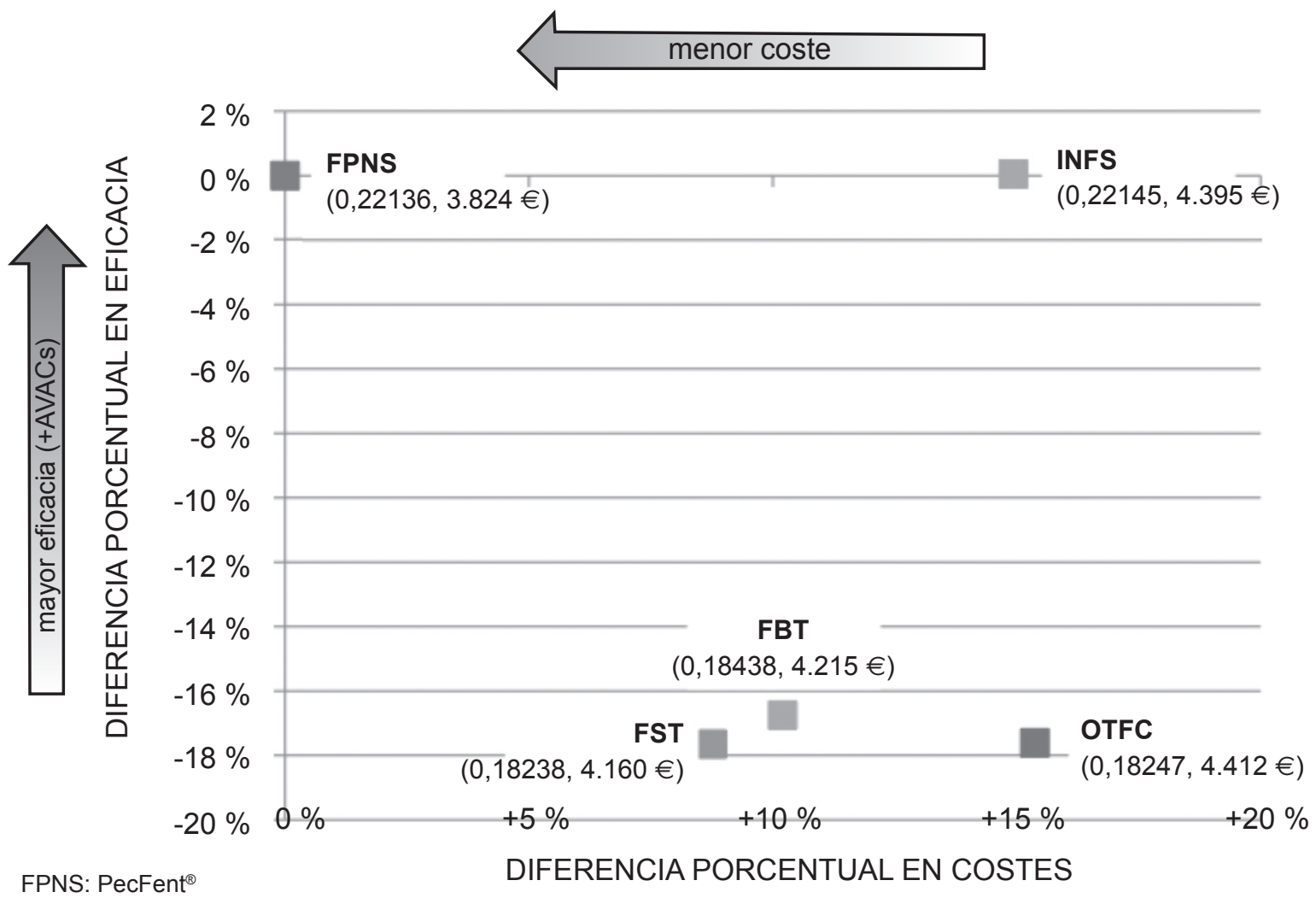

INFS: Instanyl ${ }^{\circledR}$

FBT: Effentora ${ }^{\circledR}$

La opción más efectiva (más años ganados con calidad de vida y menos costosa) fue el espray intranasal de fentanilo en pectina (FPNS). Tres de las demás opciones comparadas son inferiores en eficacia y todas presentan un mayor coste.

FST: Abstral ${ }^{\circledR}$

OTFC: Actiq $^{\circledR}$

AVAC: años ganados con calidad de vida

Fig. 3. Plano de coste/utilidad de los tratamientos farmacológicos empleados en el tratamiento del dolor irruptivo oncológico. 
posición a pagar (invertir) de $30.000 €$ por AVAC ganado (ratio coste/efectividad $<30.000 €$ ). La opción más eficiente (mayor efectividad y menor coste) en todos los casos analizados es el espray intranasal de fentanilo en pectina (FPNS).

En el análisis incremental, FPNS fue la opción dominante sobre todas las demás evaluadas: FST, OTFC, FBT e INFS.

Del análisis de los datos de nuestro estudio de modelización de coste/efectividad del espray intranasal de fentanilo en pectina FPNS se concluye:

- INFS sin proporcionar más eficacia ha demostrado ser significativamente más caro $(+15 \%)$.

- FST, OTFC, FBT han demostrado una efectividad un $18 \%$ menor que FPNS y, además, ser significativamente más caros $(+9 \% ;+15 \% ;+10 \%$, respectivamente).

\section{CORRESPONDENCIA:}

A. J. García Ruiz

Dep. Farmacología. Facultad de Medicina

29071, Málaga

e-mail: ajgr@uma.es

\section{BIBLIOGRAFÍA}

1. Davies et al. The management of cancer-related breakthrough pain: Recommendations of a task group of the Science Committee of the Association for Palliative Medicine of Great Britain and Ireland. Eur J Pain 2009;13(4):331-8.

2. Mercadante S, Maddaloni S, Roccella S, et al. Predictive factors in advance cancer pain treated only by analgesics. Pain 1992;50:151-5.

3. Faisinger et al. A multicenter study of the revised Edmonton Staging System for classifying cancer pain in advanced cancer patients. J Pain Symptom Manage 2005 Mar;29(3):224-37.

4. Portenoy R.K. et Al. Breakthrough pain: characteristics and impact in patients with cancer pain. Pain 1999;81:129-34.

5. Zeppetella G. Impact and management of breakthrough pain in cancer. Curr Opin Support Palliat Care 2009;3:1-6.

6. Davies A. et Al. Breakthrough Cancer Pain: An Observational Study of 1000 European Oncology Patients. J Pain Symptom Manage 2013 Mar 22. pii: S0885-3924(13)0020-6.

7. Zeppetella G. Evidence-based treatment of cancer-related breakthrough pain with opioids. J Natl Compr Canc Netw 2013;11(Supl. 1):S37-43.

8. Vissers D. et Al. Efficacy of intranasal fentanyl spray versus other opioids for breakthrough pain in cancer. Curr Med Res Opin 2010;26(5):1037-45.
9. Ministerio de Sanidad y Consumo. Agencia Española del Medicamento. Disponible en: http://www.aemps.gob.es/ cima/fichasTecnicas.do?metodo=detalleForm.

10. Vissers D, Nolte T, Stam WB, et al. Efficacy of intranasal fentanyl spray versus other opioids for breakthrough pain in cancer. Curr Med Res Opin 2010;26(5):1037-45.

11. Jensen MP, Karoly P, Braver S. The measurement of clinical pain intensity: A comparison of six methods. Pain 1986;27:1147-126.

12. Downie WW, Leatham PA, Rhind VM, et al. Studies with pain rating scales. Ann Rheum Dis 1978;37:378-81.

13. Kremer E, Atkinson JH, Ignelzi RJ. Measurement of pain: Patient preference does not confound pain measurement. Pain 1981;10:241-8.

14. Vissers D, Lenre M, Tolley K, Jakobsson J, Sendersky V, Jansen JP. An economic evaluation of short-acting opiods for treatment of breakthrough pain in patients with cancer. Value in Health 2011;14:274-81.

15. López-Bastida J, Oliva J, Antoñanzas F, Garcia-Altes A, Gisbert R, Mar J, Puig-Junoy J. Spanish Recommendations on Economic Evaluation of Health Technologies. European Journal of Health Economics 2010;11(5):513-20.

16. Guidelines for the Economic Evaluation of Health Technologies. Canadian Agency for Drugs and Technologies in Health (CADTH). [Consultado 7/09/2011]. Disponible en: http:// cadth.ca/en/products/methods-and-guidelines.

17. ISPOR Good Outcomes Research Practices Index. [consultado 5/09/2011]. Disponible en: http://www.ispor.org/workpaper/practices index.asp.

18. Sacristán JĀ, Oliva J, Del Llano J, et al. ¿Qué es una tecnología sanitaria eficiente en España? Gac Sanit 2002;16:334-43.

19. García Ruiz AJ, F Morata García de la Puerta, AC Montesinos Gálvez. Uso racional y eficiente del tratamiento hipocolesterolemiante. Med Clin 2008;130(7):263-6.

20. Fortner BV, Okon TA, Portenoy RK. A survey of pain-related hospitalizations, emergency department visits, and physician office visits reported by cancer patients with and without history of breakthrough pain. Journal of Pain 2002;3(1):38-44

21. Fortner BV, Demarco G, Irving G, Ashley J, Keppler G, Chavez J, et al. Description and predictors of direct and indirect costs of pain reported by cancer patients. Journal of Pain and Symptom Management 2003;25(1):9-18.

22. Abernethy AP, Wheeler JL, Fortner BV. A health economic model of breakthrough pain. American Journal of Managed Care 2008;14(5):S129-40.

23. Burton AW, Driver LC, Mendoza TR, Syed G. Oral transmucosal fentanyl citrate in the outpatient management of severe cancer pain crises: A retrospective case series. Clinical Journal of Pain 2004;20:195-7.

24. Taylor DR, Webster LR, Chun SY et al. Impact of breakthrough pain on quality of life in patients with chronic, noncancer pain: Patient perceptions and effect of treatment with oral transmucosal fentanyl citrate (OTCF, Actiq). Pain Medicine 2007;8:281-8. 